\title{
A topical issue: NMR investigations of molecular dynamics
}

\author{
Arthur G. Palmer III
}

Published online: 9 August 2009

(C) Springer Science+Business Media B.V. 2009

The 20 papers in this topical issue of the Journal of Biomolecular NMR reflect the multitude of NMR techniques and approaches used to investigate conformational dynamics critical for folding and stability, molecular recognition, and catalysis of proteins, nucleic acids, and other biological molecules. Of course, any single issue of a journal cannot include a selection of papers comprehensive enough to truly encompass the diversity of developments and applications in a field that continues to expand as rapidly and creatively as NMR spectroscopy. Many alternative selections of papers could have been made equally well and no doubt, indeed one hopes, the work described in this topical issue will inspire developments of increasingly powerful techniques and applications to increasingly deeper questions in biology.

New experimental methods drive biological investigations by NMR spectroscopy and several papers in this topical issue present new techniques or novel combinations of existing methods in solution NMR spectroscopy. The Carr-Purcell-Meiboom-Gill (CPMG) pulse sequence has emerged as a critical method for investigating conformational dynamics in biological macromolecules. Most investigations of conformational dynamics of protein backbones have used IS spin systems, such as ${ }^{1} \mathrm{H}-{ }^{15} \mathrm{~N}$ or methine ${ }^{1} \mathrm{H}-{ }^{13} \mathrm{C}$, as probes, for example, see the paper by Raleigh, Palmer and coworkers described below. To overcome this limitation, Kay and coworkers introduce new CPMG relaxation dispersion experiments for ${ }^{1} \mathrm{H}^{\alpha}$ and ${ }^{13} \mathrm{C}^{\alpha}$ spins in Gly residues (Vallurupalli et al. 2009). The

A. G. Palmer III ( $\square)$

Department of Biochemistry and Molecular Biophysics, Columbia University, 630 West 168th Street, New York, NY 10032, USA

e-mail: agp6@columbia.edu new methods are applied to a mutant of T4 lysozyme that binds hydrophobic ligands in an internal cavity in the protein. Existing theoretical analyses of relaxation during CPMG pulse trains were derived assuming infinitely short ideal refocusing pulses. Myint and Ishima provide a critical assessment of the effects of evolution and relaxation during real finite-length refocusing pulses of the CPMG pulse train (Myint and Ishima 2009). Field cycling enables measurements site-specific relaxation rate constants at low static magnetic fields with the resolution and sensitivity of a high field NMR spectrometer. Redfield, Kern, and coworkers obtain $R_{1}$ relaxation rate constants at fields as low as $4 \mathrm{~T}$ for the nucleocapsid protein of the SARS coronavirus using a home-built field cycling instrument (Clarkson et al. 2009). Together with conventional high field measurements, these data suggest correlated rigid body motions of structural motifs of the protein. Skrynnikov and coworkers use glycerol to increase solution viscosity and thereby increase the rotational diffusion correlation time of the $\alpha$-spectrin SH3 domain. The longer rotational correlation time extends the time window to which NMR relaxation methods are sensitive to internal motional processes (Xu et al. 2009). Hydrogen exchange between amide moieties and solvent is a longstanding and powerful approach for studying folding and thermodynamics of proteins. However, for large molecules, even the resolution in ${ }^{1} \mathrm{H}-{ }^{15} \mathrm{~N}$ two-dimensional correlation spectra becomes limiting and three-dimensional spectra cannot be acquired rapidly enough using conventional Cartesian sampling schemes. Wand and coworkers introduce a new method, called AMORE-HX, that uses radial sampling of a three-dimensional HNCO experiment to determine amide hydrogen exchange rates in real time (Gledhill et al. 2009). They show that radial sampling with four angles $\left(69^{\circ}, 81^{\circ}, 10^{\circ}\right.$, $27^{\circ}$ ) can resolve 328 of the 334 cross peaks that remain 
after initial dissolution of the 371-residue maltose binding protein in $\mathrm{D}_{2} \mathrm{O}$ solution, enabling exchange rates to be measured comprehensively in larger proteins. Although the majority of applications to biological molecules have used autorelaxation rate constants, such as $R_{1}$ and $R_{2}$, crosscorrelated relaxation, also called relaxation-interference, has unique properties. Weaver and Zuiderweg use longitudinal cross-correlated relaxation of amide ${ }^{1} \mathrm{H}^{\mathrm{N}}$ spins to quantify the conformational dynamics of ${ }^{1} \mathrm{H}$ spins that have dipolar coupling interactions with the ${ }^{1} \mathrm{H}^{\mathrm{N}}$ spin (Weaver and Zuiderweg 2009).

An increasing number of investigations of nucleic acid dynamics are being reported that both follow the protocols developed for proteins and utilize the unique properties of RNA and DNA. Schwalbe and coworkers use ${ }^{31} \mathrm{P}$ spin relaxation to investigate dynamics in the UUCG tetraloop, a model system that has been the subject of numerous investigations by NMR spectroscopy (Rinnenthal et al. 2009). Unexpectedly, subnanosecond dynamics are more pronounced for the phosphodiester backbone than for the ribofuranosyl and nucleobase moieties of the nucleotides. Most, but see work by Peng and coworkers described below for an exception, investigations of conformational dynamics rely on isotope enrichment of heteronuclei to increase sensitivity of (lengthy) relaxation experiments. Nikolova and Al-Hashimi introduce a method for preparing residue-specific ${ }^{13} \mathrm{C} /{ }^{15} \mathrm{~N}$-labeled elongated DNA molecules (Nikolova and Al-Hashimi 2009). They use this method to probe fast inter-helical bending motions induced by an adenine tract and identify end-fraying internal motions occurring on nanosecond timescales. As described below, solid-state NMR spectroscopy is represented in this area by a study of TAR RNA by Drobny, Varani, and coworkers.

A striking feature of this topical issue is the extent to which solid-state NMR techniques, singly or in combination with solution NMR methods, are being applied to characterize conformational dynamics of biological molecules. Methods that utilize anisotropic interactions in the solid state potentially provide critical information difficult to obtain in solution that can help constrain motional and structural models. Cady and Hong use ${ }^{1} \mathrm{H}$ rotating-frame spin-lattice relaxation, $R_{1 \rho}$, to characterize uniaxial rotational diffusion of the influenza A M2 transmembrane peptide in lipid bilayers (Cady and Hong 2009). Interestingly, the ligand amantadine both increased the membrane viscosity and improved the packing of the M2 peptide. Drobny, Varani, and coworkers use deuterium solid-state NMR to investigate motions in HIV-1 TAR RNA as a function of hydration (Olsen et al. 2009). A comparison between solid-state and solution state ${ }^{13} \mathrm{C}$ relaxation measurements indicate that ns- $\mu$ s motions become manifest as hydration approaches bulk levels. McDermott introduces two new solid-state experiments. In one paper, $\mathrm{Li}$ and
McDermott describe a new version of the CODEX experiment that utilizes dipolar couplings, which are well-known and uniform, rather than CSAs, which can vary from siteto-site in molecules (Li and McDermott 2009). In a second paper, Quinn and McDermott switch sides and use $R_{1 \rho}$ experiments to monitor reorientation of CSA tensors, allowing kinetic measurements to be made even in the absence of changes in isotropic chemical shifts (Quinn and McDermott 2009). Both of these methods are demonstrated on small molecules, but as already noted, Cady and Hong have employed ${ }^{1} \mathrm{H} R_{1 \rho}$ measurements sensitive to dipolar interactions in their study of the M2 peptide, suggesting that these new techniques will be applied quickly to biological molecules. Reif and coworkers measure ${ }^{15} \mathrm{~N} R_{1}$, ${ }^{1} \mathrm{H}-{ }^{15} \mathrm{~N}$ dipolar $/{ }^{15} \mathrm{~N}$ CSA cross-correlated relaxation, and ${ }^{1} \mathrm{H}-{ }^{15} \mathrm{~N}$ dipolar coupling constants for the $\alpha$-spectrin SH3 domain (Chevelkov et al. 2009). Importantly, the protein is highly deuterated to suppress spin diffusion and allow quantitative description of dynamics in the solid state.

A major advancement over the past decade has been the transition from a focus on development of NMR methods for characterizing conformational dynamics, generally using tractable model systems, to a focus on applications to significant biophysical and biological questions. Lipchock and Loria use CPMG relaxation dispersion measurements to delineate conformational dynamics linked to communication between active sites that are separated by $>25 \AA$ in the enzyme imidazole glycerol phosphate synthase (Lipchock and Loria 2009). Raleigh, Palmer and coworkers use ${ }^{13} \mathrm{C}^{\alpha}$ and methyl ${ }^{13} \mathrm{C}$ relaxation measurements to characterize a folding intermediate of the villin headpiece domain HP67 (O'Connell et al. 2009). In conjunction with earlier ${ }^{15} \mathrm{~N}$ relaxation measurements, the conformational dynamics of this relatively small domain are shown to be surprisingly complex. Redfield, Smith, and coworkers use ${ }^{15} \mathrm{~N} R_{2}$ and ${ }^{1} \mathrm{H}-{ }^{15} \mathrm{~N}$ RDC measurements to obtain a detailed description of the non-cooperative unfolding of the molten globule state of a variant of $\alpha$-lactalbumin that lacks disulfide bonds (Higman et al. 2009).

Considerable attention has been paid to the role of conformational entropy in ligand recognition. Akke and coworkers use ${ }^{15} \mathrm{~N}$ spin relaxation measurements to investigate dynamical changes consequent to lactose binding by the carbohydrate recognition domain of galectin-3 (Diehl et al. 2009). Peng and coworkers address the complementary problem of determining changes in flexibility of ligands and note that the effects of such changes must be known for development of accurate structure-activity relationships (Peng et al. 2009). Ligands frequently are difficult to obtain with isotopic enrichment; consequently, Peng and coworkers use natural abundance ${ }^{13} \mathrm{C}$ relaxation measurements for methylene ${ }^{13} \mathrm{CH}_{2}$ and methyl ${ }^{13} \mathrm{CH}_{3}$ groups to characterize the interaction of a 
phosphopeptide ligand with the peptidyl-prolyl isomerase, Pin1.

Spin relaxation arising from dipolar, CSA, and quadrupolar interactions is dominated by rotational diffusion of proteins in solution and internal motions near or slower than the rotational correlation time are difficult to characterize. Work by Skrynnikov described above and many applications of solid-state NMR methods are aimed at circumventing this restriction. Residual dipolar coupling constants (RDCs) provide another approach in solution for measuring conformational dynamics on time scales longer than that of rotational diffusion, the so-called supra- $\tau_{\mathrm{c}}$ regime. Extending earlier work using backbone ${ }^{1} \mathrm{H}_{-}{ }^{15} \mathrm{~N}$ RDCs, Griesinger and coworkers use methyl ${ }^{1} \mathrm{H}_{-}{ }^{13} \mathrm{C}$ RDCs to characterize supra- $\tau_{\mathrm{c}}$ motions of side chains in ubiquitin (Farès et al. 2009). These investigations are providing new understanding of the extent to which the apo states of proteins in solution sample conformations competent to bind ligands (so called selected-fit binding); parallel investigations of nucleic acid RDCs have been reported elsewhere by Al-Hashimi and others.

The explosion in NMR spectroscopic methods for studying conformational dynamics in biological molecules has been accompanied by similar developments in computer simulations, fast optical techniques, scattering and diffraction methods, and fluorescence- and force-based single molecule methods. Akke and coworkers combine computer simulations with the NMR relaxation methods already described above to investigate the influence of conformational entropy on lactose binding to the carbohydrate recognition domain of galectin-3. Brüschweiler, Blackledge, and coworkers describe improvements in reproducing ${ }^{3} \mathrm{~J}$ coupling constants using accelerated molecular dynamics simulations and simulations with long time-scale trajectories (Markwick et al. 2009). Novel field cycling measurements by Redfield, Kern, and coworkers have been described above; these investigators also use molecular dynamics simulations to assess the extent to which motions detected experimentally at specific sites in the SARS coronavirus nucleocapsid protein may be collective. Melding of these other methods with NMR spectroscopy to take advantage of the relative strengths of each technique already is providing novel insights and future advances can be anticipated.

NMR spectroscopy has been in a state of continued development since the discovery of the NMR phenomenon. Over the past 20 years, a renaissance has occurred in the range and power of spin relaxation and other approaches in NMR spectroscopy for investigation of molecular conformational dynamics. The study of larger and more complex biological systems places a premium on the experimental and theoretical capabilities of the NMR methods employed. The 20 papers in this topical issue of the Journal of
Biomolecular NMR represent the current state of the field in 2009. The next decade will bring many unanticipated and exciting developments, and the present papers will serve as guideposts to this bright future.

\section{References}

Cady SD, Hong M (2009) Effects of amantadine on the dynamics of membrane-bound influenza A M2 transmembrane peptide studied by NMR relaxation. J Biomol NMR 45:185-196

Chevelkov V, Fink U, Reif B (2009) Quantitative analysis of backbone motion in proteins using MAS solid-state NMR spectroscopy. J Biomol NMR 45:197-206

Clarkson MW, Lei M, Eisenmesser EZ, Labeikovsky W, Redfield A, Kern D (2009) Mesodynamics in the SARS nucleocapsid measured by NMR field cycling. J Biomol NMR 45:217-225

Diehl C, Genheden S, Modig K, Ryde U, Akke M (2009) Conformational entropy changes upon lactose binding to the carbohydrate recognition domain of galectin-3. J Biomol NMR 45: $157-169$

Farès C, Lakomek N-A, Walter K, Frank B, Meiler J, Becker S, Griesinger $C$ (2009) Accessing ns- $\mu$ s side chain dynamics in ubiquitin with methyl RDCs. J Biomol NMR 45:23-44

Higman VA, Rösner HI, Ugolini R, Greene LH, Redfield C, Smith LJ (2009) Probing the urea dependence of residual structure in denatured human $\alpha$-lactalbumin. J Biomol NMR 45:121-131

Gledhill JM Jr, Walters BT, Wand AJ (2009) AMORE-HX: a multidimensional optimization of radial enhanced NMRsampled hydrogen exchange. J Biomol NMR 45:233-239

Li W, McDermott AE (2009) Characterization of slow conformational dynamics in solids: dipolar CODEX. J Biomol NMR 45:227-232

Lipchock J, Loria JP (2009) Millisecond dynamics in the allosteric enzyme imidazole glycerol phosphate synthase (IGPS) from Thermotoga maritima. J Biomol NMR 45:73-84

Markwick PRL, Showalter S, Bouvignies G, Brüschweiler R, Blackledge M (2009) Structural dynamics of protein backbone $\varphi$ angles: extended molecular dynamics simulations versus experimental ${ }^{3} J$ scalar couplings. J Biomol NMR 45:17-21

Myint W, Ishima R (2009) Chemical exchange effects during refocusing pulses in constant-time CPMG relaxation dispersion experiments. J Biomol NMR 45:207-216

Nikolova EN, Al-Hashimi HM (2009) Preparation, resonance assignment, and preliminary dynamics characterization of residue specific ${ }^{13} \mathrm{C} /{ }^{15} \mathrm{~N}$-labeled elongated DNA for the study of sequence-directed dynamics by NMR. J Biomol NMR 45:9-16

O'Connell NE, Grey MJ, Tang Y, Kosuri P, Miloushev VZ, Raleigh DP, Palmer AG (2009) Partially folded equilibrium intermediate of the villin headpiece HP67 defined by ${ }^{13} \mathrm{C}$ relaxation dispersion. J Biomol NMR 45:85-98

Olsen GL, Bardaro MF Jr, Echodu DC, Drobny GP, Varani G (2009) Hydration dependent dynamics in RNA. J Biomol NMR 45:133-142

Peng JW, Wilson BD, Namanja AT (2009) Mapping the dynamics of ligand reorganization via ${ }^{13} \mathrm{CH}_{3}$ and ${ }^{13} \mathrm{CH}_{2}$ relaxation dispersion at natural abundance. J Biomol NMR 45:171-183

Quinn CM, McDermott AE (2009) Monitoring conformational dynamics with solid-state $R_{1 \rho}$ experiments. J Biomol NMR 45:5-8

Rinnenthal J, Richter C, Nozinovic S, Fürtig B, Lopez JJ, Glaubitz C, Schwalbe H (2009) RNA phosphodiester backbone dynamics of a perdeuterated cUUCGg tetraloop RNA from phosphorus-31 NMR relaxation analysis. J Biomol NMR 45:143-155 
Vallurupalli P, Hansen DF, Lundström P, Kay LE (2009) CPMG relaxation dispersion NMR experiments measuring glycine ${ }^{1} \mathrm{H}^{\alpha}$ and ${ }^{13} \mathrm{C}^{\alpha}$ chemical shifts in the 'invisible' excited states of proteins. J Biomol NMR 45:45-55

Weaver DS, Zuiderweg ERP (2009) Protein proton-proton dynamics from amide proton spin flip rates. J Biomol NMR 45:99-119
Xu J, Xue Y, Skrynnikov NR (2009) Detection of nanosecond time scale side-chain jumps in a protein dissolved in water/glycerol solvent. J Biomol NMR 45:57-72 